\title{
On the rise and fall of a ball with linear or quadratic drag
}

\author{
Peter Timmerman and Jacobus P. van der Weele ${ }^{\mathrm{a})}$ \\ Centre for Theoretical Physics, University of Twente, P.O. Box 217, 7500 AE Enschede, The Netherlands
}

(Received 29 June 1998; accepted 16 October 1998)

\begin{abstract}
We review the problem of a vertically thrown ball, with a drag force which is either linear or quadratic in the speed. It is stressed from the outset that these two types of drag correspond to specific ranges of the Reynolds number $\left(\operatorname{Re}<1\right.$ and $10^{3}<\operatorname{Re}<2 \times 10^{5}$, respectively) and do not hold outside these intervals. We also include the buoyant force in our treatment of the problem. The equations of motion are solved analytically and several true-to-life examples are discussed. The calculations are somewhat harder than for the well-known case without drag force, but no highbrow mathematics is required and the extra effort is amply compensated by the gain in realism and surprise value. (C) 1999 American Association of Physics Teachers.
\end{abstract}

\section{INTRODUCTION}

One of the classic problems of physics concerns a spherical object moving vertically through a resistive medium. One may think for instance of a cannon ball shot up into the air, or of a grain of sand sinking slowly to the bottom of a lake. If the resistive force $F(\nu)$ is either linear or quadratic in the velocity, the problem admits an analytical solution, and this will be the main topic of the present paper. First of all, however, we discuss when these two particular forms of resistive force actually occur. Both correspond to a specific interval of the Reynolds number and can be derived from the following general formula: ${ }^{1}$

$$
F(\nu)=\frac{1}{2} C_{d} \rho A \nu^{2} .
$$

Here $C_{d}$ is the so-called drag coefficient, $\rho$ the density of the medium, $A$ the object's cross-sectional area (in the case of a sphere $\pi R^{2}$ ), and $\nu$ its velocity. It should be noted that Eq. (1) is actually only valid in the case of constant $\nu$, the socalled stationary situation, and that in general (if $\nu$ varies) one should also take into account the Boussinesq-Basset viscous memory force and the "added mass" term. ${ }^{2}$ These terms are especially important if the density and the viscosity of the medium are large. On the other hand, they complicate the calculations to such a high degree that we choose to ignore them.

The most intriguing element in Eq. (1) is the drag coefficient; it depends in a complicated way on the Reynolds number $(\mathrm{Re})$, as depicted in Fig. 1. (This figure can be found in almost any textbook on hydrodynamics, see, e.g., Refs. 3 and 4.) So in order to use the above equation one first has to know the value of Re. This dimensionless number is defined as follows: ${ }^{1}$

$$
\operatorname{Re}=\frac{\rho l \nu}{\eta}
$$

where $l$ represents the characteristic length scale of the object in the cross-sectional plane (in the case of a sphere this is just the diameter $2 R$ ) and $\eta$ the dynamic viscosity of the medium.

In the hydrodynamic literature one finds several formulas to describe the curve of Fig. 1, most of which are restricted to a relatively small range of $\operatorname{Re}$ (see Refs. 4-7). An exception to this rule is the following curve-fit formula, from Ref. 4, which holds for all $0<\operatorname{Re}<2 \times 10^{5}$ :

$$
C_{d}(\mathrm{Re}) \approx \frac{24}{\mathrm{Re}}+\frac{6}{1+\sqrt{\mathrm{Re}}}+0.4
$$

In the limit for very small Reynolds number, say $\operatorname{Re}<1$, the first term dominates and we may ignore the second and third terms. With $C_{d}(\mathrm{Re})=24 / \mathrm{Re}=12 \eta / \rho R \nu$ and $A=\pi R^{2}$ the general formula in Eq. (1) then reduces to

$$
F(\nu)=6 \pi \eta R \nu
$$

This is known as Stokes' formula. It tells us that the drag on a (very) slowly moving sphere is linearly proportional to its velocity. The formula applies to the sinking grain of sand mentioned above (we shall come back to this example in Sec. II), to micro-organisms in water, ${ }^{8}$ or to tiny dust particles floating in air. Generally speaking, though, the condition $\operatorname{Re}<1$ is rather restrictive and rarely met in practice. This has not prevented the linear drag force or "viscous friction," with its appealing simplicity and nice theoretical properties (e.g., it can be incorporated in the Lagrangian formalism ${ }^{9}$ ), from becoming the favorite type of damping in the physics literature.

The second interval we focus upon is $10^{3}<\operatorname{Re}<2 \times 10^{5}$, where the drag coefficient is seen [from Fig. 1 or Eq. (3)] to be approximately constant: $C_{d} \approx 0.4$. In that case Eq. (1) reduces to

$$
F(\nu)=0.2 \rho \pi R^{2} \nu^{2} .
$$

So in this regime the drag on the sphere is quadratic in the velocity. It applies for instance to a pebble dropped from the Leaning Tower of Pisa (except for a fraction of a second at the very start, see also Sec. III) or a sky diver and is commonly known as "air drag.",

As mentioned before, for the above two forms of the drag force the problem of a vertically thrown ball can be solved analytically. In practice, of course, one often goes outside the domains of linear $(\operatorname{Re}<1)$ and quadratic drag $\left(10^{3}<\operatorname{Re}<2\right.$ $\left.\times 10^{5}\right)$. For instance, for a bullet shot upwards with an initial Reynolds number of $10^{6}$, Re will pass through every value from $10^{6}$ to zero, at the top of the trajectory. Nevertheless, in certain cases the drag force remains linear or quadratic during (almost) the whole trajectory and we restrict ourselves to these. It should be noted that several of the calculations to be presented can be found elsewhere, scattered throughout the 


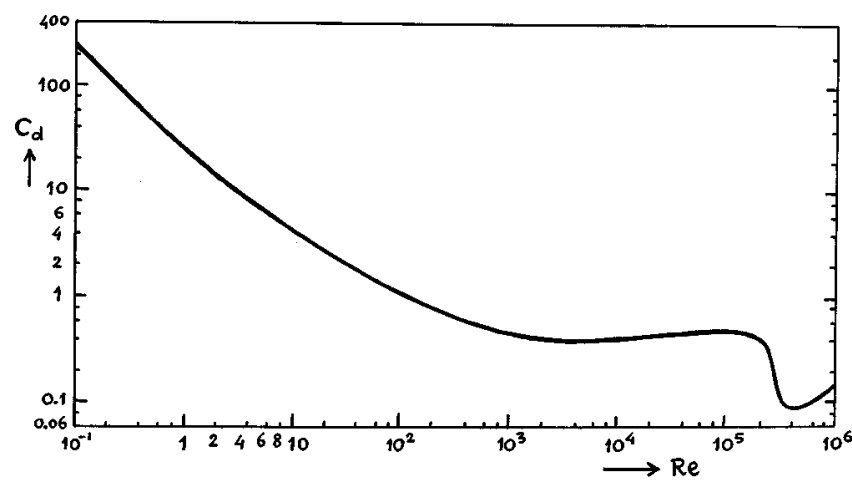

Fig. 1. The drag coefficient $C_{d}$ of a sphere as a function of the Reynolds number Re. Both $C_{d}$ and Re are dimensionless numbers. Mind the logarithmic scales. The sudden drop in $C_{d}$ at $\operatorname{Re} \approx 2.5 \times 10^{5}$ (the so-called drag crisis) is associated with the change of the boundary layer on the sphere's surface from laminar to turbulent.

volumes of this journal, but in our opinion there are at least three good reasons for putting this subject on the stage once more.

First, we shall pay proper attention to the domains of validity of the linear and quadratic drag and discuss typical examples of each. There is some need for this, since in the literature on this subject the physical problem often seems to be only an excuse for a nice mathematical exercise.

Second, we include the buoyant force, which is almost always forgotten or ignored in the literature on this topic. This is somewhat curious, since the medium that is responsible for the drag force at the same time introduces a buoyant force.

Third, by carrying out all calculations step by step we hope to make them palatable for students, and perhaps bring the subject to the classroom here and there. In textbook problems on projectile motion the air resistance is habitually ignored, and this has led to a widespread belief that inclusion of resistive forces would make any of these problems intractable. The two cases to be considered provide welcome counterexamples.

The paper is built up as follows. Linear drag is discussed in Sec. II, quadratic drag in Sec. III, and in Sec. IV we make some concluding remarks. Throughout the paper we shall often compare our results with those for the simple case without any drag, and (hence) also without buoyancy. Let us therefore briefly recall it here. In the absence of drag and buoyancy a ball thrown up from ground level with initial speed $\nu_{0}$ has velocity:

$$
\nu(t)=\nu_{0}-g t,
$$

and its height is given by

$$
h(t)=\nu_{0} t-\frac{1}{2} g t^{2} .
$$

It immediately follows that

$$
t_{\text {up }}=t_{\text {down }}=\frac{\nu_{0}}{g},
$$

and that the ball reaches a height of

$$
h_{\max }=\frac{\nu_{0}^{2}}{2 g} \text {. }
$$

Furthermore, it is clear that the ball hits the ground with exactly the same velocity $\nu_{0}$ as with which it started. All this will be changed by the drag (and buoyant) forces. It is perhaps a good idea, before reading on, to try to predict how the various quantities will be changed. A few simple experiments may help. For instance, will the time to go up still be equal to the time to come down? Will the total time ( $t_{\text {up }}$ $\left.+t_{\text {down }}\right)$ be longer or shorter than $2 \nu_{0} / g$ ? And how much will the final velocity differ from $\nu_{0}$ ? We shall come back to these questions in due course.

\section{LINEAR DRAG}

The equation of motion, along a vertical path, for a sphere with linear drag [as in Eq. (4)] reads:

$$
m \frac{d \nu}{d t}=-\left(m-m^{*}\right) g-6 \pi \eta R \nu .
$$

Here $m^{*} g$, with $m^{*}$ the mass of the displaced medium, is the buoyant force. The above equation may also be written as:

$$
\frac{d \nu}{d t}=-\left(1-m^{*} / m\right) g-\frac{6 \pi \eta R}{m} \nu
$$

or equivalently:

$$
\frac{d \nu}{d t}=-\widetilde{g}-\alpha \nu
$$

where $\widetilde{g}$ (the effective gravitational acceleration) is given by

$$
\tilde{g}=\left(1-m^{*} / m\right) g=\left(1-\rho / \rho_{\text {ball }}\right) g
$$

and, with $m=\frac{4}{3} \pi R^{3} \rho_{\text {ball }}$,

$$
\alpha=\frac{6 \pi \eta R}{m}=\frac{9 \eta}{2 R^{2} \rho_{\text {ball }}} .
$$

Equation (12) is readily integrated [with initial condition $\left.\nu(0)=\nu_{0}\right]$ to give the velocity:

$$
\nu(t)=\left(\nu_{0}+\widetilde{g} / \alpha\right) e^{-\alpha t}-\frac{\widetilde{g}}{\alpha} .
$$

This looks quite different from the solution without drag [given by Eq. (6)] but in the limit for $\alpha \rightarrow 0$ and $\widetilde{g} \rightarrow g$ it nevertheless reduces to it, as it should; this may be checked by a glance at the Taylor expansion [Eq. (52)] in the Appendix. Equation (15) shows that the velocity during the downward journey cannot grow beyond $-\widetilde{g} / \alpha$, the so-called terminal velocity:

$$
\nu_{\text {term }}=-\frac{\tilde{g}}{\alpha}=-\frac{2\left(\rho_{\text {ball }}-\rho\right) g R^{2}}{9 \eta} .
$$

This also follows directly from Eq. (12), when $d \nu / d t$ is set equal to zero.

Integrating Eq. (15) yields the height $h(t)$ :

$$
h(t)=\int \nu(t) d t=-\frac{\left(\nu_{0}+\widetilde{g} / \alpha\right)}{\alpha} e^{-\alpha t}-\frac{\widetilde{g}}{\alpha} t+c_{1} .
$$

The integration constant $c_{1}$ is determined from the initial condition $h(0)=0$, and we get:

$$
h(t)=\frac{\widetilde{g}}{\alpha^{2}}\left(1+\frac{\alpha \nu_{0}}{\widetilde{g}}\right)\left(1-e^{-\alpha t}\right)-\frac{\widetilde{g}}{\alpha} t .
$$

Again, this solution reduces to its frictionless counterpart [Eq. (7)] in the limit $\alpha \rightarrow 0$ and $\widetilde{g} \rightarrow g$, as exemplified by the 
Taylor expansion [Eq. (53)] in the Appendix.

Given the above expressions for the velocity and the height we now go on to determine the quantities $t_{\text {up }}, h_{\max }$, and $t_{\text {down }}$. The main reason why we choose just these three quantities is that they are easy to measure, and may thus be checked in relatively simple experiments. The time to go up is found by setting $\nu(t)$ equal to zero and thus, from Eq. (15),

$$
t_{\text {up }}=\frac{1}{\alpha} \ln \left(1+\frac{\alpha \nu_{0}}{\widetilde{g}}\right)=\left(\frac{\nu_{0}}{\widetilde{g}}\right)-\frac{\alpha}{2}\left(\frac{\nu_{0}}{\widetilde{g}}\right)^{2}+\frac{\alpha^{2}}{3}\left(\frac{\nu_{0}}{\widetilde{g}}\right)^{3}-\cdots .
$$

The Taylor expansion is given to show how $t_{\mathrm{up}}$ approaches its frictionless counterpart in the limit $\left(\alpha \nu_{0} / \widetilde{g}\right) \downarrow 0$. It converges only for $\left(\alpha \nu_{0} / \widetilde{g}\right) \leqslant 1$, but that is all right, since for finite values of $\left(\alpha \nu_{0} / \widetilde{g}\right)$ one naturally uses the exact expression.

The maximum height is obtained by substituting $t_{\text {up }}$ in the equation for $h(t)$ :

$h_{\max }=\frac{\nu_{0}}{\alpha}-\frac{\widetilde{g}}{\alpha^{2}} \ln \left(1+\frac{\alpha \nu_{0}}{\widetilde{g}}\right)=\frac{\nu_{0}^{2}}{2 \widetilde{g}}-\alpha \frac{\nu_{0}^{3}}{3 \widetilde{g}^{2}}+\alpha^{2} \frac{\nu_{0}^{4}}{4 \widetilde{g}^{3}}-\cdots$,

and the above remarks concerning the Taylor expansion also apply here. Finally, the time to go down is determined by setting $h(t)=0$. This yields the following transcendental equation:

$$
\alpha t=\left(1+\frac{\alpha \nu_{0}}{\widetilde{g}}\right)\left(1-e^{-\alpha t}\right),
$$

with two solutions, $t=0$ and $t=t_{\text {total }}=t_{\text {up }}+t_{\text {down }}$. Unfortunately, the latter cannot be given explicitly, but the following elegant relation derived by Lekner ${ }^{10}$ is a worthy alternative:

$$
t_{\text {total }}=\frac{\nu_{0}+\left|\nu_{\text {hit }}\right|}{\widetilde{g}} .
$$

One might object that it just transfers the pain to $\nu_{\text {hit }}$, for which we also have no explicit solution. We do know, however, that $\left|\nu_{\text {hit }}\right|<\nu_{0}$ (this follows simply from the fact that the ball has given up energy to the resistive medium) and therefore that $t_{\text {total }}<2 \nu_{0} / \widetilde{g}$. This may also be checked by inserting $t=2 \nu_{0} / \widetilde{g}$ in the formula for $h(t)$. Supposing that $\left.\alpha t=2 \alpha \nu_{0} / \widetilde{g}\right) \ll 1$, and using the Taylor expansion Eq. (53), this yields $h\left(2 \nu_{0} / \widetilde{g}\right)=-\alpha \cdot\left(2 \nu_{0}^{3} / 3 \widetilde{g}^{3}\right)+\cdots$, which is negative, indicating that the ground must be hit before time $2 \nu_{0} / \widetilde{g}$. In the literature, where the buoyant force is habitually forgotten (and where $\widetilde{g}$ is therefore taken to be equal to $g$ ) it is inferred in the same stroke that $t_{\text {total }}$ is always smaller than the total time in the absence of drag, i.e., smaller than $2 \nu_{0} / g$ (see, e.g., Ref. 10). Of course, this is not generally true. If $\widetilde{g}$ is only slightly less than $g$ (i.e., if the buoyancy plays a minor role), the total time as given by Eq. (22) will indeed be smaller than $2 \nu_{0} / g$, but in situations where $\widetilde{g}$ deviates sufficiently from $g$ it will be larger. In the next section, below Eq. (44), we will come back to this.

The value of $\widetilde{g}$ may even be negative, for instance for an air bubble in water, and in that case there is no descent. The bubble just keeps going upwards (mathematically speaking forever, physically speaking until it reaches the surface) with a velocity that approaches the value $-\widetilde{g} / \alpha$, which is now positive.

In the same vein it can be checked that the way down takes longer than the way up. This can be done, for instance, by inserting $t=2 t_{\text {up }}$ in the expression for $h(t)$ :

$$
\begin{aligned}
h\left(2 t_{\mathrm{up}}\right) & =\frac{\widetilde{g}}{\alpha^{2}}\left[\left(1+\frac{\alpha \nu_{0}}{\widetilde{g}}\right)-\left(1+\frac{\alpha \nu_{0}}{\widetilde{g}}\right)^{-1}-2 \ln \left(1+\frac{\alpha \nu_{0}}{\widetilde{g}}\right)\right] \\
& =\alpha \frac{\nu_{0}^{3}}{3 \widetilde{g}^{2}}-\alpha^{2} \frac{\nu_{0}^{4}}{2 \widetilde{g}^{3}}+\cdots .
\end{aligned}
$$

Again, in performing the Taylor expansion, we have assumed that $\left(\alpha \nu_{0} / \widetilde{g}\right)<1$. The expression for $h\left(2 t_{\text {up }}\right)$ then yields a positive value, so at time $2 t_{\text {up }}$ the ball is still on its way down, or in other words: $t_{\text {down }}>t_{\text {up }}$. The same result can also be inferred more generally (see Ref. 11) and even without performing any calculation: ${ }^{12}$ during its motion the ball continuously gives up energy to the medium. So the kinetic energy, and hence the velocity, at any given height is less on the way down than on the way up (the potential energy being the same). It follows that also the average velocity on the way down is smaller than the average velocity on the way up, and consequently the descent takes longer than the ascent.

The argument can also be given in terms of forces. ${ }^{13}$ During the ascent the resistive force and (effective) gravity act in the same downward direction while during the descent they are opposed to each other, resulting in a smaller net force. So the acceleration is smaller during the descent, which therefore takes more time.

Let us now consider the example mentioned in Sec. I, a grain of sand slowly sinking to the bottom of a lake. In this case $\rho_{\text {ball }}=2.67 \times 10^{3} \mathrm{~kg} / \mathrm{m}^{3}$, while for the medium (water at room temperature) we have $\rho=1.00 \times 10^{3} \mathrm{~kg} / \mathrm{m}^{3}$ and $\eta$ $=1.00 \times 10^{-3} \mathrm{~kg} / \mathrm{m} \mathrm{s}$. The terminal velocity is calculated from Eq. (16):

$$
\nu_{\text {term }}=-3.64 \times 10^{6} R^{2} \quad(\text { in } \mathrm{m} / \mathrm{s}) .
$$

With this velocity the Reynolds number becomes

$$
\operatorname{Re}=\frac{2 \rho R \nu_{\text {term }}}{\eta}=7.28 \times 10^{12} R^{3},
$$

which means that only sufficiently small grains with radius $R<0.5 \times 10^{-4} \mathrm{~m}$ (that is, a diameter of one-tenth of a millimeter) will fall according to Stokes' law $(\operatorname{Re}<1)$. Take for instance a grain of sand with $R=0.2 \times 10^{-4} \mathrm{~m}$ which is released from rest. Its velocity is given by Eq. (15) with $\nu_{0}$ $=0$ :

$$
\nu(t)=-\frac{\widetilde{g}}{\alpha}\left(1-e^{-\alpha t}\right)=-1.46 \times 10^{-3}\left(1-e^{-4.2 \times 10^{3} t}\right) .
$$

The terminal velocity, 1.46 millimeters per second, is attained almost instantaneously; after $t=\ln 100 /\left(4.2 \times 10^{3}\right)$ $=1.1 \times 10^{-3} \mathrm{~s}(1 \mathrm{~ms})$, it is already approached within $1 \%$. So we may safely say that the grain sinks all the way to the bottom with the terminal velocity. If the lake is $3 \mathrm{~m}$ deep, this will take $34 \mathrm{~min}$. In the absence of the buoyant force we would have found $21 \mathrm{~min}$, indicating once again that this force is not to be ignored.

Now, in order to illustrate the equations derived in this section, we take this same grain of sand and toss it upwards 

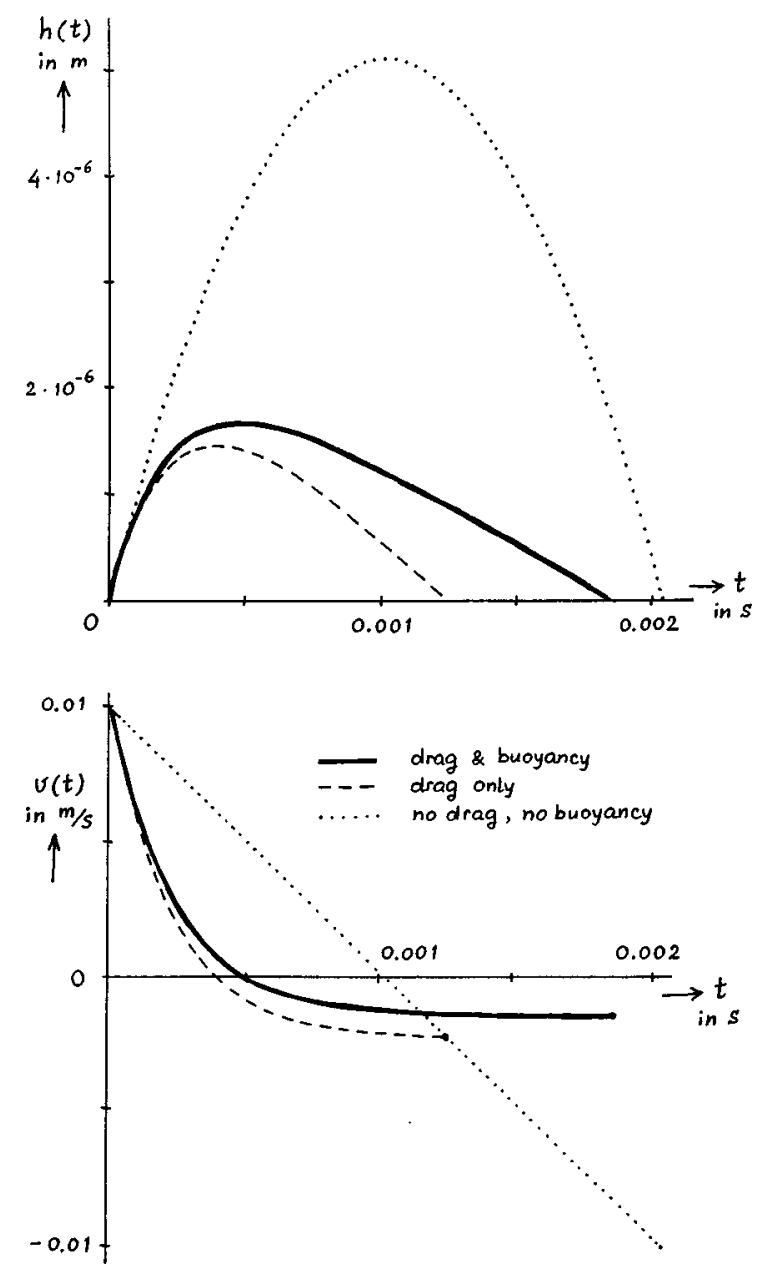

Fig. 2. The height $h(t)$ and velocity $\nu(t)$ of the grain of sand discussed in the text, shot upwards in water with an initial velocity of $0.01 \mathrm{~m} / \mathrm{s}$. The solid curves are the "true" ones, including the effects of both drag and buoyancy (with $\alpha=4213 \mathrm{~s}^{-1}$ and $\widetilde{g}=6.14 \mathrm{~m} / \mathrm{s}^{2}$ ). In the dashed curves the buoyancy is neglected $\left(\alpha=4213 \mathrm{~s}^{-1}, \widetilde{g}=g=9.81 \mathrm{~m} / \mathrm{s}^{2}\right)$; in the dotted curves both buoyancy and drag are neglected (i.e., $\alpha=0 \mathrm{~s}^{-1}$ and $\widetilde{g}=g=9.81 \mathrm{~m} / \mathrm{s}^{2}$ ).

from the bottom of the lake (a rather fancy experiment) and determine its height $h(t)$ and velocity $\nu(t)$. To keep $\operatorname{Re}<1$ we have to choose a small initial velocity; we set $\nu_{0}$ $=0.01 \mathrm{~m} / \mathrm{s}$. The result is depicted in Fig. 2. The dotted curves represent the simple case without drag or buoyancy; the dashed curves show the case when drag is taken into account, but buoyancy is not (i.e., when $\widetilde{g}$ is taken equal to $g=9.81 \mathrm{~m} / \mathrm{s}^{2}$ ); finally, the solid curves are the "true" ones, including the effects of both drag and buoyancy (with $\widetilde{g}$ $=6.14 \mathrm{~m} / \mathrm{s}^{2}$ ).

The first thing to be noted is, of course, the extreme smallness of the times and distances involved, showing once again that the applicability of Stokes' law is limited to microscopic phenomena. For instance, the grain of sand rises only over $1.6 \times 10^{-6} \mathrm{~m}$, less than one-tenth of its radius. Nevertheless, the figure brings out very clearly that (in the presence of drag) the way down takes longer than the way up, and also that the grain of sand very quickly approaches its terminal velocity $\left(-1.46 \times 10^{-3} \mathrm{~m} / \mathrm{s}\right)$. Another interesting thing is that the dashed velocity curve stops exactly at the point where it meets the dotted velocity. This is a graphical illustration of Eq. (22), since in the case that $\widetilde{g}=g$ this equation tells us that $\nu_{\text {hit }}=\nu_{0}-g t_{\text {total }}$, and this coincides precisely (at $\left.t=t_{\text {total }}\right)$ with the velocity of the frictionless case: $\nu(t)=\nu_{0}$ $-g t$. We further note that the dashed and dotted velocity curves go through zero with the same slope (namely $-g$ ), but that the slope of the solid curve is different $(-\widetilde{g})$.

\section{QUADRATIC DRAG}

Whereas in the case of linear drag, the way up and the way down constituted one problem (one differential equation) with one solution, in the case of quadratic drag they give rise to two separate problems. This is because $\nu^{2}$ does not change sign in going from the way up to the way down; hence, to ensure that the quadratic drag force opposes the motion we have to insert the correct signs ourselves. This means that we get two different equations of motion, which have to be treated separately. It should be noted that the mathematical gist of this section has appeared earlier in this journal in the form of a problem; ${ }^{14}$ the downward part alone has been described in Refs. 15-17. The new thing is that we have included the buoyant force. It does not make the calculations any harder, so there is really no reason to avoid this force.

During the upward journey the drag force [given by Eq. (5)] acts in the downward direction, and the equation of motion takes the form:

$$
m \frac{d \nu_{\uparrow}}{d t}=-\left(m-m^{*}\right) g-0.2 \rho \pi R^{2} \nu_{\uparrow}^{2} .
$$

This equation can be rewritten as follows:

$$
\frac{d \nu_{\uparrow}}{d t}=-\widetilde{g}\left(1+\gamma^{2} \nu_{\uparrow}^{2}\right),
$$

where $\gamma=\sqrt{0.2 \rho \pi R^{2} / m \widetilde{g}}=\sqrt{0.15\left(\rho / \rho_{\text {ball }}\right) / R \widetilde{g}}$. Integrating and inserting the initial condition $\nu_{\uparrow}(0)=\nu_{0}$ yields:

$$
\nu_{\uparrow}(t)=\frac{1}{\gamma} \tan \left(-\gamma \widetilde{g} t+\arctan \gamma \nu_{0}\right)
$$

From this expression immediately follows the time when the ball reaches its maximum height [i.e., when $\nu_{\uparrow}(t)$ becomes zero]:

$$
t_{\mathrm{up}}=\frac{1}{\gamma \widetilde{g}} \arctan \gamma \nu_{0}=\frac{\nu_{0}}{\widetilde{g}}-\gamma^{2} \frac{\nu_{0}^{3}}{3 \widetilde{g}}+\gamma^{4} \frac{\nu_{0}^{5}}{5 \widetilde{g}}-\cdots
$$

Here, as always, the Taylor expansion is meant to indicate how the result approaches the frictionless expression. One should not use it for $\gamma \nu_{0}>1$, because then the series diverges. Integrating the velocity gives the height of the rising ball:

$$
\begin{aligned}
h_{\uparrow}(t) & =\int \nu_{\uparrow}(t) d t \\
& =\frac{1}{\gamma} \cdot \frac{1}{\gamma \widetilde{g}} \ln \left[\cos \left(-\gamma \widetilde{g} t+\arctan \gamma \nu_{0}\right)\right]+c_{2},
\end{aligned}
$$

where the integration constant $c_{2}$ is determined from the initial condition $h_{\uparrow}(0)=0$ :

$$
c_{2}=\frac{-1}{\gamma^{2} \widetilde{g}} \ln \left[\cos \left(\arctan \gamma \nu_{0}\right)\right]=\frac{1}{\gamma^{2} \widetilde{g}} \ln \sqrt{1+\left(\gamma \nu_{0}\right)^{2}} .
$$


This integration constant is not only elegantly simple, but also significant. Its elegance stems from the relation $\cos (\arctan \alpha)=1 / \sqrt{1+\alpha^{2}}$. Its significance lies in the fact that it happens to be the maximum height $h_{\max }$, that is to say,

$$
\begin{aligned}
h_{\max }=\frac{1}{\gamma^{2} \widetilde{g}} \ln \sqrt{1+\left(\gamma \nu_{0}\right)^{2}} & =\frac{1}{2 \gamma^{2} \widetilde{g}} \ln \left[1+\left(\gamma \nu_{0}\right)^{2}\right] \\
& =\frac{\nu_{0}^{2}}{2 \widetilde{g}}-\gamma^{2} \frac{\nu_{0}^{4}}{4 \widetilde{g}}+\gamma^{4} \frac{\nu_{0}^{6}}{6 \widetilde{g}}-\cdots .
\end{aligned}
$$

Of course, the Taylor expansion is only valid for $\gamma \nu_{0} \leqslant 1$. From the above equations it is seen that $h_{\uparrow}(t)$ is built up as $h_{\text {max }}$ plus a negative term that decreases with time. Putting the pieces together we get:

$$
\begin{aligned}
h_{\uparrow}(t) & =h_{\max }+\frac{1}{\gamma^{2} \widetilde{g}} \ln \left[\cos \left(-\gamma \widetilde{g} t+\arctan \gamma \nu_{0}\right)\right] \\
& =\frac{1}{\gamma^{2} \widetilde{g}} \ln \left[\sqrt{1+\left(\gamma \nu_{0}\right)^{2}} \cos \left(-\gamma \widetilde{g} t+\arctan \gamma \nu_{0}\right)\right] .
\end{aligned}
$$

When we get near the top of the trajectory, our analysis is, strictly speaking, no longer valid; the Reynolds number drops below $10^{3}$ and the drag force is no longer quadratic in the speed. However, under normal circumstances this is such a tiny part of the trajectory that we do not have to worry about it. In air, with $\rho=1.293 \mathrm{~kg} / \mathrm{m}^{3}$ and $\eta=17.1$ $\times 10^{-6} \mathrm{~kg} /(\mathrm{m} \mathrm{s})$, the Reynolds number, given by Eq. (2), is

$$
\mathrm{Re}=151.2 \times 10^{3} R \nu .
$$

For a pebble with radius $R=1 \mathrm{~cm}$ this means that $\nu$ should exceed $0.66 \mathrm{~m} / \mathrm{s}$ for the quadratic drag to apply. In the frictionless case this speed is already attained after $0.07 \mathrm{~s}$, when the pebble is $2.2 \mathrm{~cm}$ under the top. In the presence of friction these values will be of the same order of magnitude.

We therefore ignore the subtleties around the top and turn directly to the downward part of the trajectory. Here the drag force and the gravitational force act in opposite directions and the equation of motion takes the form:

$$
\frac{d \nu_{\downarrow}}{d t}=-\widetilde{g}\left(1-\gamma^{2} \nu_{\downarrow}^{2}\right) .
$$

This equation has an extra minus sign as compared with the upward equation, and as a consequence we now get a tanh solution instead of a tan (this may be traced back to the difference between $\cosh ^{2} x-\sinh ^{2} x=1$ and $\cos ^{2} x+\sin ^{2} x$ $=1$ ). To be specific, the solution reads:

$$
\nu_{\downarrow}(t)=-\frac{1}{\gamma} \tanh \gamma \widetilde{g}\left(t-t_{\text {up }}\right) .
$$

From this expression it is seen that the coefficient $\gamma$ is not just a cosmetic factor to keep the formulae transparent but also has a physical meaning: $\nu_{\downarrow}(t)$ can never exceed the terminal velocity

$$
\nu_{\text {term }}=-\frac{1}{\gamma}=-\sqrt{\frac{m \widetilde{g}}{0.2 \rho \pi R^{2}}} .
$$

This terminal velocity can also be inferred directly from Eq. (36), when $d \nu_{\downarrow} / d t$ is set equal to zero. With $m=\frac{4}{3} \pi R^{3} \rho_{\text {ball }}$ it can be written as:

$$
\nu_{\text {term }}=-\sqrt{6.67\left(\frac{\rho_{\text {ball }}}{\rho}-1\right) g R},
$$

which for $g=9.81 \mathrm{~m} / \mathrm{s}^{2}$ and $\rho=1.293 \mathrm{~kg} / \mathrm{m}^{3}$ (air) reduces to $\nu_{\text {term }}=-8.1 \sqrt{\left(\left(\rho_{\text {ball }} / 1.293\right)-1\right) R}$. This shows, for instance, that if two balls are dropped simultaneously from the Leaning Tower of Pisa they will not hit the ground at the same time unless they have equal values of $\left(\left(\rho_{\text {ball }} / 1.293\right)-1\right) R$. In this context the following passage from Galileo's Dialogues Concerning Two New Sciences, quoted in Ref. 15, is very interesting: "Aristotle says that "an iron ball of one hundred pounds falling from a height of one hundred cubits reaches the ground before a one-pound ball has fallen a single cubit.' I say that they arrive at the same time. You find, on making the experiment, that the larger outstrips the smaller by two finger-breadths, that is, when the larger has reached the ground, the other is short of it by two fingerbreadths." This passage shows two things. First, that Galileo was well aware that the simultaneous landing for which he has become famous is in fact an idealization. Second, it shows that Galileo was a rather sloppy experimenter or had very thick fingers, for the difference can be calculated to be no less than a meter; it is fair to note, though, that the experimental verification of this is not at all easy since the difference in time is only $1 / 30$ of a second. ${ }^{15}$ For those who want to check these numbers we mention that 100 cubits is about $60 \mathrm{~m}$ and that $\rho_{\text {iron }}=7.87 \times 10^{3} \mathrm{~kg} / \mathrm{m}^{3}$.

Resuming the calculation at hand and integrating the velocity given by Eq. (37), we get the height of the falling ball:

$$
h_{\downarrow}(t)=\int \nu_{\downarrow}(t) d t=h_{\text {max }}-\frac{1}{\gamma^{2} \widetilde{g}} \ln \cosh \gamma \widetilde{g}\left(t-t_{\text {up }}\right) .
$$

The structure of this formula resembles that of $h_{\uparrow}(t)$; it is built up as $h_{\max }$ minus a distance, which in this case increases with time. Whereas the distance in the case of $h_{\uparrow}(t)$ was of the form $\ln (\cos )$, in the present case it has the form $\ln (\cosh )$. With $h_{\max }$ as in Eq. (33) we get:

$$
h_{\downarrow}(t)=\frac{1}{\gamma^{2} \widetilde{g}} \ln \left(\frac{\sqrt{1+\left(\gamma \nu_{0}\right)^{2}}}{\cosh \gamma \widetilde{g}\left(t-t_{\text {up }}\right)}\right) \text {. }
$$

We have now completed the calculation of the velocity and the height as functions of $t$, and in passing we have also determined the times of ascent $\left(t_{\text {up }}\right)$ and the maximal height $\left(h_{\max }\right)$. Another quantity of interest is the time of descent $\left(t_{\text {down }}\right)$. To calculate this we set $h_{\downarrow}(t)$ equal to zero, that is,

$$
\cosh \gamma \widetilde{g} t_{\text {down }}=\sqrt{1+\left(\gamma \nu_{0}\right)^{2}},
$$

from which it follows that

$$
\begin{aligned}
t_{\text {down }} & =\frac{1}{\gamma \widetilde{g}} \operatorname{arccosh} \sqrt{1+\left(\gamma \nu_{0}\right)^{2}} \\
& =\frac{1}{\gamma \widetilde{g}} \operatorname{arcsinh} \gamma \nu_{0}=\frac{1}{\gamma \widetilde{g}} \ln \left(\gamma \nu_{0}+\sqrt{1+\left(\gamma \nu_{0}\right)^{2}}\right) \\
& =\frac{\nu_{0}}{\widetilde{g}}-\gamma^{2} \frac{\nu_{0}^{3}}{6 \widetilde{g}}+\gamma^{4} \frac{3 \nu_{0}^{5}}{40 \widetilde{g}}+\cdots .
\end{aligned}
$$

Remarkably, this quantity which could not be solved analytically in the case of the allegedly simpler linear drag, here comes out without any difficulty. Comparing the Taylor expansions of $t_{\text {up }}$ and $t_{\text {down }}$ (assuming that they converge, i.e., 
that $\gamma \nu_{0} \leqslant 1$ ), one immediately sees that $t_{\text {down }}>t_{\text {up }}$. This is just as in Sec. II for linear drag, and indeed, the arguments given there [below Eq. (23)] apply equally well to the present case. In the literature, when $\widetilde{g}$ is always taken to be equal to $g$, it is inferred from Eq. (43) that $t_{\text {down }}$ can never become larger than $\nu_{0} / g$ (the time of descent in the absence of dissipation) and hence that the total time $t_{\text {up }}+t_{\text {down }}$ is always smaller than its frictionless counterpart $2 \nu_{0} / g$. However,

$$
\begin{aligned}
t_{\text {total }} & =\frac{1}{\gamma \widetilde{g}}\left\{\arctan \gamma \nu_{0}+\ln \left(\gamma \nu_{0}+\sqrt{1+\left(\gamma \nu_{0}\right)^{2}}\right\}\right. \\
& =\frac{2 \nu_{0}}{\widetilde{g}}-\gamma^{2} \frac{\nu_{0}^{3}}{2 \widetilde{g}}+\gamma^{4} \cdot \frac{11 \nu_{0}^{5}}{40 \widetilde{g}}+\cdots
\end{aligned}
$$

and this can very well exceed the value $2 \nu_{0} / g$, if the buoyancy is significant. Take for instance a light children's ball with radius $15 \mathrm{~cm}$, such that the mass of the displaced air is $m^{*}=18.3 \mathrm{~g}$. Putting the ball on a letter balance one finds a mass $\left(m-m^{*}\right)$ of $60 \mathrm{~g}$, say, so the proper mass of the ball (plastic plus air inside) is $m=78.3 \mathrm{~g}$. The corresponding value of $\widetilde{g}$ is $7.52 \mathrm{~m} / \mathrm{s}^{2}$, and $\gamma=0.176 \mathrm{~s} / \mathrm{m}$. If one throws this ball upwards with a velocity of $\nu_{0}=5.0 \mathrm{~m} / \mathrm{s}$ the total time of flight according to Eq. (44) is $1.15 \mathrm{~s}$ (with $t_{\text {up }}=0.55 \mathrm{~s}$ and $t_{\text {down }}=0.60 \mathrm{~s}$ ), which is larger than $2 \nu_{0} / g=1.02 \mathrm{~s}$. This is illustrated in Fig. 3.

By inserting $t_{\text {down }}$ in the expression for the downward velocity, the speed with which the ball hits the ground is now easily calculated:

$$
\begin{aligned}
\nu_{\text {hit }}=\nu_{\downarrow}\left(t_{\text {total }}\right) & =-\frac{1}{\gamma} \tanh \left(\operatorname{arcsinh} \gamma \nu_{0}\right) \\
& =-\frac{\nu_{0}}{\sqrt{1+\left(\gamma \nu_{0}\right)^{2}}} .
\end{aligned}
$$

This is a surprisingly charming result, taking into account the somewhat cumbersome expressions that went into it. One can immediately check that it has the right limiting behavior for $\gamma=0$ : The final velocity is then equal to $-\nu_{0}$. Also the limit for $\gamma \nu_{0} \rightarrow \infty$ is correct, since $\nu_{\text {hit }}$ then approaches the terminal velocity $-1 / \gamma$. In a practical situation, with a given ball and medium (and thus a certain fixed value of $\gamma$ ), one can come very close to this limit by making $\nu_{0}$ as large as possible.

In Fig. 3, the end of the dashed curve lies almost on the dotted line, and from this one might get the impression that Eq. (22), which was derived for linear drag, is still valid (cf. our discussion of Fig. 2). However, with the above expression for $\nu_{\text {hit }}$, one easily checks that $t_{\text {total }}$ [Eq. (44)] is not the same as

$$
\frac{\nu_{0}+\left|\nu_{\text {hit }}\right|}{\widetilde{g}}=\frac{2 \nu_{0}}{\widetilde{g}}-\gamma^{2} \frac{\nu_{0}^{3}}{2 \widetilde{g}}+\gamma^{4} \frac{3 \nu_{0}^{5}}{8 \widetilde{g}}+\cdots .
$$

For the children's ball the difference is small, since $\gamma \ll 1$ and the value of $\nu_{0}$ is modest, but in other instances (see, for example, Fig. 4) the difference may be quite substantial. In any case, Eq. (22) is not exact for quadratic drag.

From Eq. (45) we can also infer how much of the ball's energy has been dissipated during the flight. At the moment it hits the ground its kinetic energy is
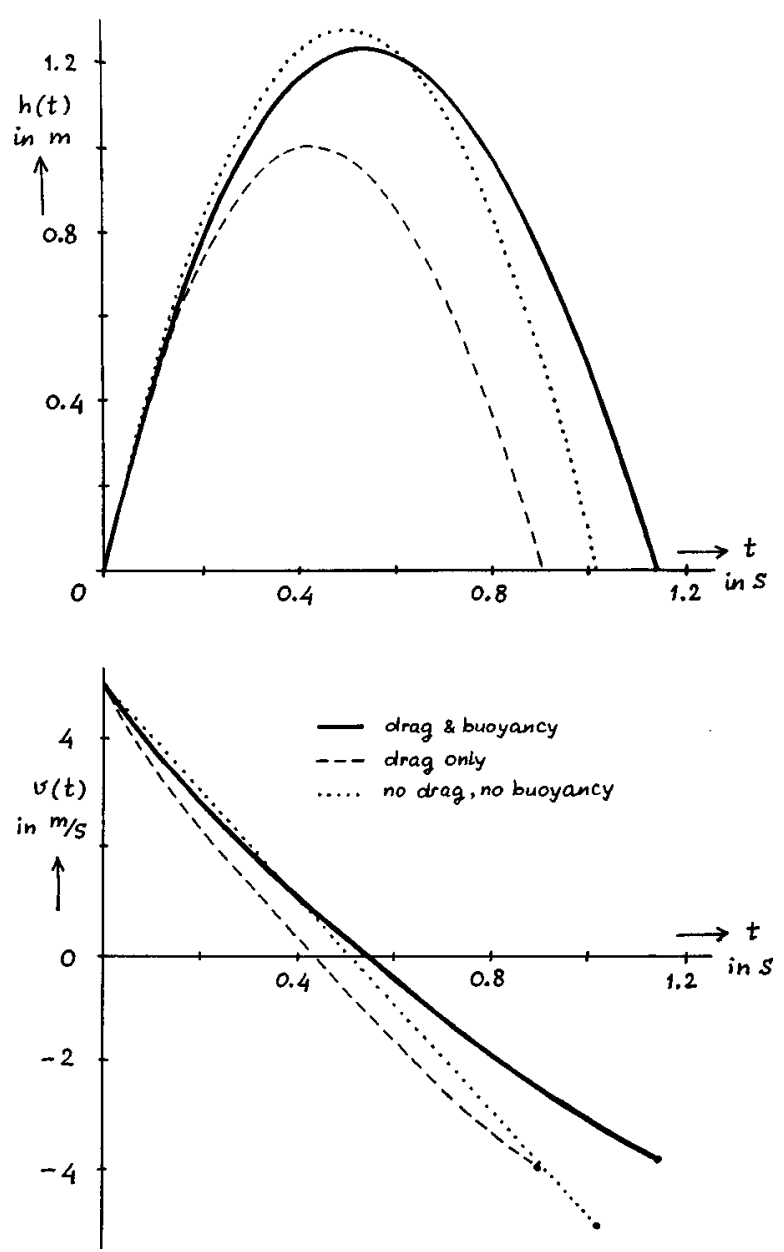

Fig. 3. The height $h(t)$ and velocity $\nu(t)$ for the children's ball discussed in the text. The solid curves correspond to the actual ball, with air drag and buoyancy (with $\gamma=0.176 \mathrm{~s} / \mathrm{m}$ and $\tilde{g}=7.52 \mathrm{~m} / \mathrm{s}^{2}$ ). In the dashed curves the buoyancy is omitted (i.e., we have taken $\widetilde{g}=g=9.81 \mathrm{~m} / \mathrm{s}^{2}$ and hence $\gamma$ $=0.154 \mathrm{~s} / \mathrm{m}$ ), and the dotted curves represent the simple case without either drag or buoyancy $\left(\gamma=0 \mathrm{~s} / \mathrm{m}\right.$ and $\tilde{g}=g=9.81 \mathrm{~m} / \mathrm{s}^{2}$ ). Note that $t_{\text {total }}$ for the solid curve is larger than for the dotted one.

$$
E_{\mathrm{kin}}=\frac{1}{2} m \frac{\nu_{0}^{2}}{1+\left(\gamma \nu_{0}\right)^{2}},
$$

which is a factor $1+\left(\gamma \nu_{0}\right)^{2}$ smaller than the initial kinetic energy. Thus for $\gamma=0$ there is no loss of energy, as it should, and for any finite value of $\gamma$ the energy loss increases with growing initial velocity $\nu_{0}$.

Let us see how all this works out for a spherical bullet shot upwards with an initial velocity $\nu_{0}=150 \mathrm{~m} / \mathrm{s}$. We take the bullet to be made of lead $\left(\rho_{\text {lead }}=11.3 \times 10^{3} \mathrm{~kg} / \mathrm{m}^{3}\right)$ and to have a mass of $12 \mathrm{~g}$, so that its radius is $0.63 \mathrm{~cm}$. The value of $\widetilde{g}$ is then $0.9999 g$ (so the buoyant force is quite negligible) and the terminal speed $-1 / \gamma$ is, according to Eq. (39),

$$
\nu_{\text {term }}=-60.1 \mathrm{~m} / \mathrm{s} \quad(=-216 \mathrm{~km} / \mathrm{h}) \text {. }
$$

The associated value of $\gamma \nu_{0}$ is 2.5 and hence, according to Eq. (45), the bullet hits the ground with almost the terminal velocity, namely with $55.7 \mathrm{~m} / \mathrm{s}$. And with $1+\left(\gamma \nu_{0}\right)^{2}=7.25$ it follows that no less than $86.2 \%$ of the initial energy is lost to the air. Also the other characteristic quantities are greatly affected. Whereas the bullet in the absence of air resistance 

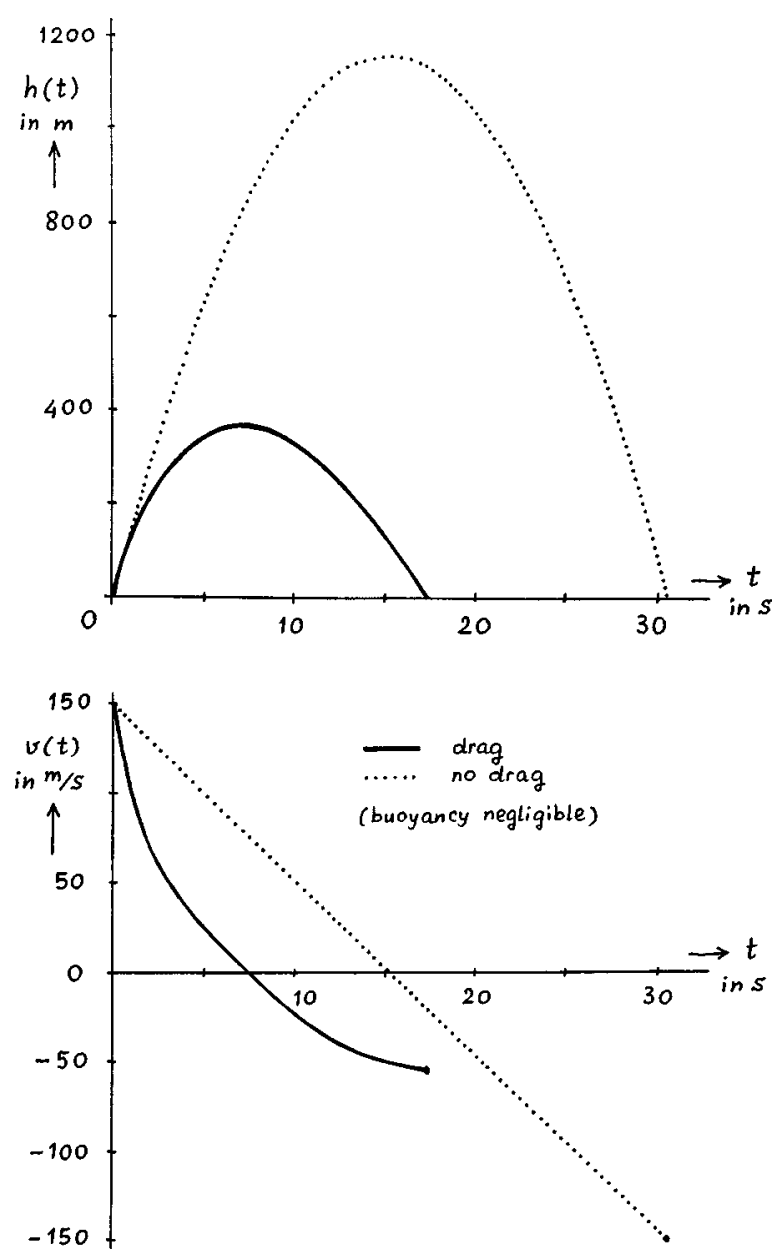

Fig. 4. The height $h(t)$ and velocity $\nu(t)$ for the spherical bullet discussed in the text. The solid curves are for the actual bullet $(\gamma=0.0167 \mathrm{~s} / \mathrm{m})$, while the dotted curves represent the case without air drag $(\gamma=0 \mathrm{~s} / \mathrm{m})$. The buoyant force is negligible in this example, so $\widetilde{g} \approx g=9.81 \mathrm{~m} / \mathrm{s}^{2}$.

would reach a height of $1.15 \mathrm{~km}$ [see Eq. (9)], it now does not go higher than $0.36 \mathrm{~km}$. As for the time of flight: instead of $2 \times 15.3 \mathrm{~s}=30.6 \mathrm{~s}$ without air resistance, we now get $t_{\text {up }}$ $=7.3 \mathrm{~s}$ and $t_{\text {down }}=10.1 \mathrm{~s}$, adding up to a mere $17.4 \mathrm{~s}$. These values can also be read off from Fig. 4, where we have depicted $h(t)$ and $\nu(t)$ for the bullet as functions of $t$. The dotted curves represent the case without air drag.

The above example is related to a question that came up during the recent disturbances in Albania, where the people made it a habit to crowd together and shoot bullets straight up into the air. Reporters in the field were somewhat concerned about this. The issue was discussed on television and in several newspapers, and in particular the speed with which the bullets would hit the ground (or an unfortunate bystander) was a hot topic. The bullets considered were 10-15 $\mathrm{g}$, and fired by a Kalashnikov rifle, which means that the initial velocity was about $700 \mathrm{~m} / \mathrm{s}$. In one newspaper the bullets were estimated to return to the earth with the speed of sound $(330 \mathrm{~m} / \mathrm{s})$, another newspaper reported that the bullets came down with the same speed as with which they left the rifle $(700 \mathrm{~m} / \mathrm{s})$, and a third one claimed that the impact velocity was no less than a smashing $120000 \mathrm{~m} / \mathrm{s}$. The latter two values are clearly false, illustrating how poor our intuition for air resistance really is, but even the first one is considerably faster than the terminal velocity $(60 \mathrm{~m} / \mathrm{s})$ of our spherical bullet above. Note that in our example we took $\nu_{0}=150 \mathrm{~m} / \mathrm{s}$ instead of $700 \mathrm{~m} / \mathrm{s}$ (in order to keep $\operatorname{Re}<2$ $\times 10^{5}$ and stay within the domain of quadratic drag) but, although it brings about large changes in the height and time of flight, this has no effect on the terminal velocity.

Now one might object that bullets are usually not spherical but torpedo shaped, and that the value of $1 / \gamma$ for the latter is somewhat larger since its drag coefficient is only about $C_{d}$ $=0.30$ (against 0.4 for a sphere). But this is not sufficient to bridge the gap, since for a torpedo-shaped bullet of $12 \mathrm{~g}$ one would get $1 / \gamma \sim 90 \mathrm{~m} / \mathrm{s}$ and this is still much smaller than the speed of sound. Moreover, the torpedo shape prevents the bullet from falling straight (rather, it comes tumbling down) and this means that its translational velocity will always remain significantly smaller than $1 / \gamma$, in no way does it get near the speeds reported in the newspapers.

As a final example, let us have one more look at the children's ball mentioned under Eq. (44). We drop it from a height $h_{0}=30 \mathrm{~m}$, and assume that it falls straight down (ignoring the fact that in reality the ball will always start to wobble as a consequence of the formation of von Kármán vortices). Disregarding the very short initial stage for which $\nu<0.044 \mathrm{~m} / \mathrm{s}$, when the Reynolds number has not yet reached the value $10^{3}$ [cf. Eq. (35)], its velocity is given by Eq. (37) with $t_{\text {up }}=0 \mathrm{~s}$ :

$$
\nu_{\downarrow}(t)=-\frac{1}{\gamma} \tanh \gamma \widetilde{g} t=-5.67 \tanh (1.32 t) .
$$

The terminal velocity of $5.67 \mathrm{~m} / \mathrm{s}$ is approached within $1 \%$ after $2.0 \mathrm{~s}$. The altitude of the ball is then

$$
\begin{aligned}
h_{\downarrow}(t)=h_{0}-\frac{1}{\gamma^{2} \widetilde{g}} \ln \cosh \gamma \widetilde{g} t & =30-4.28 \ln \cosh (1.32 t) \\
& =21.6 \mathrm{~m} .
\end{aligned}
$$

The rest of the way down takes $21.6 / 5.67=3.8 \mathrm{~s}$, so the ball reaches the ground after $5.8 \mathrm{~s}$. If the experiment were performed in a vacuum tube, i.e., in the absence of drag and buoyancy, one would find $t=\sqrt{2 h_{0} / g}=2.5 \mathrm{~s}$.

It may be noted that the time of descent also follows from Eq. (43), if we assume (for the sake of the argument) that the height of $30 \mathrm{~m}$ was acquired as a result of a tremendous throw. We then first determine the associated value of $\nu_{0}$ by setting $h_{0}=h_{\max }$, i.e., $30=4.28 \ln \sqrt{1+\left(\nu_{0} / 5.67\right)^{2}}$. This yields a value $\nu_{0}=6245 \mathrm{~m} / \mathrm{s}(!)$ and inserting this in Eq. (43) we get $t_{\text {down }}=5.8 \mathrm{~s}$ again.

\section{CONCLUDING REMARKS}

We have now come to the end of the paper and a few concluding remarks are in order. First, we have restricted ourselves to resistive forces which depend either linearly or quadratically on the velocity. These cases correspond, as discussed in Sec. I, to $\operatorname{Re}<1$ and $10^{3}<\operatorname{Re}<2 \times 10^{5}$, respectively. Outside these intervals our calculations do not apply and one should turn to a computer. ${ }^{18}$ Some of the qualitative conclusions of the present paper, however, remain valid also in the general case. For instance, the way down always takes longer than the way up $\left(t_{\text {down }}>t_{\text {up }}\right)$.

Second, we have included the buoyant force, which means that we deal with an effective gravitational acceleration $\widetilde{g}$ rather than with $g$ itself. We have restricted ourselves to 
cases where $\widetilde{g}$ remains positive; if $\widetilde{g}$ were made negative (as for a helium-filled balloon) the ball would go up all right, but never come down. In the literature the buoyant force is commonly disregarded (which means that one always deals with $g$ instead of $\widetilde{g}$ ), and in that case one can show that for all resistive forces $F(\nu) \propto \nu^{n}$ with $n \geqslant 1$ the total time of flight is smaller than the frictionless time $2 \nu_{0} / g .{ }^{10} \mathrm{We}$ have seen that in reality the matter is more subtle and that the total time of flight can very well exceed $2 \nu_{0} / g$.

Third, we have restricted ourselves to purely vertical trajectories. In practice, of course, a slight horizontal component can hardly be ruled out, but this will not have a great influence on quantities like the maximum height and the final velocity. In the case of linear drag the now two-dimensional problem still admits an analytical solution. One simply takes the solution for the horizontal component of the motion,

$$
\begin{aligned}
& \nu_{x}(t)=\nu_{0 x} e^{-\alpha t}, \\
& x(t)=\frac{\nu_{0 x}}{\alpha}\left(1-e^{-\alpha t}\right)
\end{aligned}
$$

and superimposes this on the solution for the vertical component, given by Eqs. (15) and (18). The result is a curved path which in the limit for $\alpha \rightarrow 0$ (and $\widetilde{g} \rightarrow g$, as far as the vertical component is concerned) tends to the well-known textbook parabola. The quadratic drag is a harder nut to crack, even though the equation of motion in the horizontal direction can again be solved analytically; the difficulty is that this time a linear superposition is not allowed, since the system is nonlinear. How the problem should be handled in this case is described in Refs. 19-21.

In the context of nonvertical trajectories, there has recently been a lively discussion in this journal (Refs. 22-24) about the optimum angle of projection, i.e., the angle under which one should launch a ball in order to achieve the greatest range. In vacuum this angle is $45^{\circ}$, and it is also well known that in the presence of air drag it is somewhat less than $45^{\circ}$. That is, optimal shots in air are "low." The same is true in the case of linear drag, and indeed, one might be tempted to think that it holds for any conceivable drag force $F(\nu)$ $\propto \nu^{n}$, but this is not the case. In Ref. 23 it was shown that if the exponent $n$ exceeds some critical value (around 3.5, depending on the drag coefficient) the optimum angle can very well be a few degrees larger than $45^{\circ}$. It is fascinating to see that such a venerable topic can still have surprises in store.

The fourth and final remark is that we have treated the gravitational acceleration and the density of the medium as constants. For the situations described in the present paper this is certainly a good approximation but it rules out, for instance, trajectories that reach very high into the atmosphere or even escape into space. ${ }^{25}$ An interesting account of a fall from high altitude can be found in Ref. 26.

\section{ACKNOWLEDGMENTS}

We would like to thank G. J. de Bruin, I. C. C. de Bruin, and G. L. Wiersma for carefully reading the manuscript and for their valuable remarks.

\section{APPENDIX: TAYLOR EXPANSIONS OF THE VELOCITIES AND HEIGHTS}

In this Appendix we give the Taylor expansions for $\nu(t)$ and $h(t)$ in the presence of drag and buoyancy. They are derived from the exact analytical solutions in the main text and elucidate the behavior for small drag. In the limit $\alpha$ $\rightarrow 0$ (for linear drag) and $\gamma^{2} \rightarrow 0$ (for quadratic drag) only the first term is retained and this term is equal to the frictionless quantities given by Eqs. (6) and (7). In taking these limits, $\widetilde{g}$ is simultaneously replaced by $g$, since the absence of drag also implies absence of buoyancy.

In the case of linear drag the exact expression for the velocity is given by Eq. (15). Its Taylor expansion reads:

$\nu(t)=\left(\nu_{0}-\widetilde{g} t\right)-\alpha t\left(\nu_{0}-\frac{1}{2} \widetilde{g} t\right)+\alpha^{2} \frac{1}{2} t^{2}\left(\nu_{0}-\frac{1}{3} \widetilde{g} t\right)-\cdots$,

which for $\alpha \rightarrow 0$ and $\widetilde{g} \rightarrow g$ indeed reduces to the frictionless velocity [Eq. (6)]. The height, given by Eq. (18) in the main text, has the following expansion:

$$
\begin{aligned}
h(t)= & \left(\nu_{0} t-\frac{1}{2} \widetilde{g} t^{2}\right)-\alpha \frac{1}{2} t\left(\nu_{0} t-\frac{1}{3} \widetilde{g} t^{2}\right) \\
& +\alpha^{2} \frac{1}{6} t^{2}\left(\nu_{0} t-\frac{1}{4} \widetilde{g} t^{2}\right)-\cdots
\end{aligned}
$$

and, again, in the limit $\alpha \rightarrow 0$ and $\widetilde{g} \rightarrow g$ this reduces to its frictionless counterpart [Eq. (7)] as it should.

Likewise, in the case of quadratic drag we get for the way up:

$$
\begin{aligned}
& \nu_{\uparrow}(t)=\left(\nu_{0}-\widetilde{g} t\right)-\gamma^{2} \widetilde{g} t\left(\nu_{0}^{2}-\nu_{0} \widetilde{g} t+\frac{1}{3} \widetilde{g}^{2} t^{2}\right)+\cdots, \\
& h_{\uparrow}(t)=\left(\nu_{0} t-\frac{1}{2} \widetilde{g} t^{2}\right)-\gamma^{2} \frac{1}{12} \widetilde{g} t^{2}\left(6 \nu_{0}^{2}-4 \nu_{0} \widetilde{g} t+\widetilde{g}^{2} t^{2}\right)+\cdots
\end{aligned}
$$

and for the way down:

$$
\begin{aligned}
\nu_{\downarrow}(t)= & \left(\nu_{0}-\widetilde{g} t\right)-\gamma^{2}\left(\frac{2}{3} \nu_{0}^{3}-\nu_{0}^{2} \widetilde{g} t+\nu_{0} \widetilde{g}^{2} t^{2}-\frac{1}{3} \widetilde{g}^{3} t^{3}\right) \\
& +\cdots \\
h_{\downarrow}(t)= & \left(\nu_{0} t-\frac{1}{2} \widetilde{g} t^{2}\right)-\gamma^{2} \frac{1}{12 \widetilde{g}}\left(2 \nu_{0}^{4}-8 \nu_{0}^{3} \widetilde{g} t+6 \nu_{0}^{2} \widetilde{g}^{2} t^{2}\right. \\
& \left.-4 \nu_{0} \widetilde{g}^{3} t^{3}+\widetilde{g}^{4} t^{4}\right)+\cdots .
\end{aligned}
$$

${ }^{\text {a) }}$ Author to whom all correspondence should be addressed.

${ }^{1}$ See, for instance, L. D. Landau and E. M. Lifshitz, Fluid Mechanics (Oxford U.P., New York, 1959).

${ }^{2} \mathrm{C}$. Pozridikis, Introduction to Theoretical and Computational Fluid Dynamics (Oxford U.P., New York, 1997), p. 311.

${ }^{3}$ R. W. Fox and A. T. McDonald, Introduction to Fluid Mechanics (Wiley, New York, 1992), 4th ed., p. 461.

${ }^{4}$ F. M. White, Viscous Fluid Flow (McGraw-Hill, New York, 1974), pp. 206-210

${ }^{5}$ H. L. Dryden, F. D. Murnaghan, and H. Bateman, Hydrodynamics (Dover, New York, 1956), pp. 316-317.

${ }^{6} \mathrm{M}$. van Dyke, Perturbation Methods in Fluid Mechanics (Academic, New York, 1964), p. 149.

${ }^{7}$ F. S. Sherman, Viscous Flow (McGraw-Hill, New York, 1990), pp. $288-$ 295.

${ }^{8}$ E. M. Purcell, "Life at low Reynolds number,' Am. J. Phys. 45, 3-11 (1977).

${ }^{9}$ This is done by means of the Rayleigh dissipation function, see, e.g., E. T. Whittaker, A Treatise on the Analytical Dynamics of Particles and Rigid Bodies (Cambridge, U.P., New York, 1970), pp. 230-232.

${ }^{10}$ J. Lekner, "What goes up must come down; will air resistance make it return sooner, or later?'” Math. Magazine 55, 26-28 (1982).

${ }^{11}$ J.-M. Lévy-Leblond, "Problem and solution to the problem on page 15," Am. J. Phys. 51, 15 (1983); 51, 88 (1983).

${ }^{12}$ E. Mendis, "Letter to the Editor," Am. J. Phys. 50, 680 (1982).

${ }^{13}$ E. A. Phillips, "Letter to the Editor," Am. J. Phys. 50, 583 (1982).

${ }^{14} \mathrm{~A}$. Tan, "Problem and solution to the problem on page 147,' Am. J. Phys. 50, 147 (1982); 50, 189 (1982). 
${ }^{15}$ G. Feinberg, "Fall of bodies near the earth," Am. J. Phys. 33, 501-502 (1964).

${ }^{16}$ J. Lindemuth, "The effect of air resistance on falling balls," Am. J. Phys. 39, 757-759 (1971).

${ }^{17}$ B. L. Coulter and C. G. Adler, "Can a body pass a body falling through the air?" Am. J. Phys. 47, 841-846 (1979).

${ }^{18}$ Also before the advent of the computer interesting things have been said about the general case, for instance by Newton (who treats a combination of linear and quadratic drag in part II of his Principia), the Bernoulli brothers (who considered drag forces with an arbitrary power of the velocity), and D'Alembert [who showed that the following four forms of the drag force admit an analytical solution: $F(\nu)=a+b \nu^{n}, \quad F(\nu)=a$ $+b \ln \nu, F(\nu)=a \nu^{n}+R+b \nu^{-n}$, and $F(\nu)=a(\ln \nu)^{n}+R \ln \nu+b$, see Ref. 9, p. 230].

${ }^{19}$ J. M. J. Kooy and J. W. H. Uytenbogaart, Ballistics of the Future (De Technische Uitgeverij H. Stam, Haarlem, 1946), pp. 118-131; here all cases of $\nu^{n}$ drag up to $n=5$ are considered.

${ }^{20} \mathrm{G}$. W. Parker, "Projectile motion with air resistance quadratic in the speed," Am. J. Phys. 45, 606-610 (1977).
${ }^{21} \mathrm{H}$. Erlichson, "Maximum projectile range with drag and lift, with particular application to golf," Am. J. Phys. 51, 357-362 (1983).

${ }^{22} \mathrm{C}$. W. Groetsch, "On the optimal angle of projection in general media," Am. J. Phys. 65, 797-799 (1997).

${ }^{23}$ R. H. Price and J. D. Romano, "Aim high and go far-Optimal projectile launch angles greater than $45^{\circ}$," Am. J. Phys. 66, 109-113 (1998).

${ }^{24}$ R. H. Price and J. D. Romano, "Comment on 'On the optimal angle of projection in general media' by C. W. Groetsch [Am. J. Phys. 65 (8), 797-799 (1997)],'’ Am. J. Phys. 66, 114 (1998).

${ }^{25}$ Such throws are beyond the scope of the paper also in another respect: They require tremendous initial velocities which cause the Reynolds number to go far beyond $2 \times 10^{5}$. (In fact, this number is already exceeded by bullets from an ordinary rifle, which have an initial velocity of about $700 \mathrm{~m} / \mathrm{s}$ ). In practice, of course, one does not catapult the projectile in one stroke but equips it with some kind of motor to propel it during its flight.

${ }^{26}$ P. Mohazzabi and J. H. Shea, "High-altitude free fall," Am. J. Phys. 64, 1242-1246 (1996).

\section{TOE}

I suggest that contemporary physicists' obsession with a theory of everything is socially irresponsible. In expecting society to provide billions of dollars to support this quest, TOE physicists have become like a decadent priesthood, demanding that the populace build them ever more elaborate cathedrals, with spires reaching ever higher into their idea of heaven. Since a theory of everything would be not only utterly irrelevant to daily human life and concerns, but also incomprehensible to the vast majority of people, TOE physicists can be likened to the late medieval Scholastics. This is the twentieth-century equivalent of asking how many angels could dance on the head of a pin.

Margaret Wertheim, Pythagoras' Trousers—God, Physics, and the Gender Wars (Random House, New York, 1995), p. 14. 